\section{Alimentação de crianças no primeiro semestre de vida: enfoque no aleitamento materno exclusivo}

\section{Children in the first semester of life: focus on exclusive breastfeeding}

Vera Lúcia Fugita dos Santos 1

Zaida Aurora Sperli Geraldes Soler 2

Reinaldo Azoubel 3
1,3 Programa de Pós-graduação em Ciências da Saúde. Faculdade de Medicina. Av. Brigadeiro Faria Lima, 5416. São José do Rio Preto, SP, Brasil. CEP 15.090-000 E-mail: verafugita@hotmail.com

2 Diretoria de Extensão Curricular. Faculdade de Medicina de São José do Rio Preto, SP, Brasil.

\begin{abstract}
Objectives: describe the type of diet offered to 205 children in the first six months of life.

Methods: descriptive exploratory study, focusing on exclusive breastfeeding in the first six months of life. Survey was performed in the city of Votuporanga, interior of São Paulo, during the national day of multiple vaccination.

Results: the majority of mothers breastfed their children, but less than half of them exclusively in their first six months of life. The majority of respondents were between 15 and 31 years old; were married or lived with their companions; had incomplete elementary education level and family income ranged from one to six minimum salaries. $62.6 \%$ of the women were oriented on breastfeeding during prenatal care.

Conclusions: research results determined the value of surveying the diet offered to newborn children in their first semester of life; as well as the need to orient women throughout all of their reproductive cycle to have them adhere to exclusive breastfeeding.

Key words Breast feeding, Feeding
\end{abstract}

\section{Resumo}

Objetivos: descrever o tipo de alimentação recebida por 205 crianças nos primeiros seis meses de vida.

Métodos: estudo descritivo, de caráter exploratório, enfocando-se o aleitamento materno exclusivo. A pesquisa foi realizada na cidade de Votuporanga, interior de São Paulo, durante um dia nacional de multivacinação. As informações foram obtidas por meio de entrevista estruturada com as mães das crianças incluídas no estudo.

Resultados: a maioria das mães amamentou, mas menos da metade realizou aleitamento materno exclusivo nos primeiros seis meses de vida do bebê. A maioria das entrevistadas tinham idade entre 15 e 31 anos; eram casadas ou viviam em união consensual; com grau de escolaridade em nível fundamental incompleto e com rendimento familiar de um até seis salários mínimos. 62,6\% das mulheres receberam orientação sobre a amamentação durante o pré-natal.

Conclusões: os resultados desta pesquisa apontam para a importância da investigação do tipo de alimento oferecido para a criança durante o primeiro semestre de vida; assim como para a necessidade de orientação das mulheres durante todo o ciclo reprodutivo, a fim de obtermos maior aderência ao aleitamento materno exclusivo.

Palavras-chave Alimentação, Aleitamento materno 


\section{Introdução}

Nos últimos anos têm sido freqüentes as reuniões científicas, discussões e debates sobre a alimentação de crianças no primeiro semestre de vida, sendo destacados os benefícios proporcionados pelo aleitamento natural exclusivo, em especial nos países em desenvolvimento, onde a sobrevivência infantil muitas vezes depende da condição do bebê ser amamentado ou não.

Vários estudos revelam a importância do leite humano na redução da morbi-mortalidade infantil, por suas propriedades como fonte de alimento, de afetividade e de proteção contra doenças. Tais condições são suficientes para que especialistas do mundo inteiro recomendem a amamentação exclusiva até o sexto mês de vida do bebê, complementando com outros alimentos até os dois anos. ${ }^{1}$

Além de água, vitaminas e sais minerais, o leite materno contém imunoglobinas, algumas enzimas e lisozimas e muitos outros fatores que ajudam a proteger a criança contra infecções, incluindo-se anticorpos, hormônios e outros componentes que não estão presentes em outras fórmulas infantis de leite.

Os programas de incentivo ao aleitamento materno exclusivo fazem parte de uma experiência realizada em 1990, na Itália, idealizada pela Organização Mundial da Saúde (OMS) e o Fundo das Nações Unidas para a Infância (UNICEF), cujo objetivo foi mobilizar profissionais de saúde e autoridades para mudanças nas rotinas das instituições de saúde, visando prevenir o desmame precoce. Contando com a participação do Brasil, nesse encontro foi elaborado o documento "Declaração de Inoccenti", contendo um conjunto de metas para a prática da amamentação de forma exclusiva até os quatro ou seis meses de vida, e com a introdução de alimentos complementares até o segundo ano de vida ou mais. Ainda nesse encontro foi idealizada a "Iniciativa Hospital Amigo da Criança", que inclui os 10 passos para orientar gestantes sobre os benefícios da amamentação e das desvantagens do uso de substitutos de leite materno. 4,5

Com o objetivo de reafirmar as metas da "Declaração de Inoccenti", os organismos internacionais lançaram em 2002 a "Estratégia Global sobre Alimentação Infantil e da Criança Pequena" (GSIYCF), aprovada como resolução da Assembléia Mundial de Saúde, na OMS, ocorrida no mesmo ano, que incluiu a questão de como trabalhar para atingir tais metas entre as mães HIV positivas e famílias em situações especiais. 6

No Brasil, a partir da década de 80 , foram propostas várias estratégias visando o aumento da prevalência do aleitamento materno, verificando-se que houve incremento nesses índices. ${ }^{7}$ No entanto, esse incremento não é uniforme, demonstrando que pesquisas sobre tendências locais do padrão de amamentação são fundamentais, pois avaliam os serviços e embasam mudanças e ajustes nas práticas de promoção e incentivo ao aleitamento materno. 6,8

Com a proposta de descentralização do Sistema Único de Saúde (SUS), cabe aos municípios a responsabilidade pelo planejamento, execução e monitoramento de suas ações de saúde. Nesse contexto, notou-se que os "Dias Nacionais de Multivacinação" (DNM) são considerados propícios para a realização de avaliação de programas de saúde no enfoque da amamentação, por sua ampla cobertura, praticidade, baixo custo e confiabilidade. Além disso, pesquisas dessa natureza permitem desvelar aspectos da amamentação e nutrição do lactente, que propiciam ações de vigilância à saúde e acompanhamento nos serviços de saúde. 7,9

Ante o exposto, o objetivo desta pesquisa, realizada em um "Dia Nacional de Multivacinação" em um município do interior do Estado de São Paulo, é descrever o tipo de alimentação fornecida a crianças durante os primeiros seis meses de vida, enfocandose o aleitamento materno exclusivo nessa população.

\section{Métodos}

Este é um estudo de natureza descritiva, realizado em Votuporanga por ocasião do "Dia Nacional de Multivacinação", em 25 de agosto de 2001, relacionada a 205 crianças menores de um ano. Foram preservados os aspectos éticos de pesquisas envolvendo seres humanos, com a apreciação e aprovação do projeto de pesquisa por um Comitê de Ética em Pesquisa e pelo consentimento esclarecido das mães/cuidadores respondentes.

Esclarece-se que Votuporanga é um município localizado na região Noroeste do Estado de São Paulo, Brasil, a $500 \mathrm{~km}$ da capital, com população estimada em 76.000 habitantes. Tem como principais atividades econômicas o comércio, a indústria moveleira e agropecuária, dispondo de três hospitais e 10 Postos de Atendimento (PAS).

Para obter as informações sobre o tipo de alimentação recebida por crianças nos primeiros seis meses de vida, realizou-se uma entrevista com mães ou cuidadores de crianças até um ano de vida selecionadas aleatoriamente, que compareceram aos 19 postos de vacinação instalados no município. Foi utilizado um formulário estruturado, contendo 
questões pertinentes à caracterização sóciodemográfica da mãe (idade, estado civil, escolaridade e renda familiar) e da criança (sexo, data de nascimento e peso ao nascer); dados sobre a gestação, parto e puerpério (pré-natal, intercorrências no parto e puerpério); dados sobre a amamentação (tipo, intercorrências nas mamas, "pega adequada", uso de chupeta e interferências familiares).

Para o pré-teste do formulário, foi selecionada aleatoriamente uma das Unidades Básicas de Saúde do município, onde ocorrem reuniões semanais do grupo de apoio e incentivo ao aleitamento materno. Essa atividade aconteceu na semana que antecedeu a pesquisa, teve a participação de 18 mães que estavam presentes no dia, as quais foram previamente esclarecidas pela pesquisadora sobre o trabalho à ser desenvolvido. Nessa oportunidade foi realizado o treinamento dos 34 acadêmicos do sexto período do curso de enfermagem que, em duplas ou individualmente, abordaram e entrevistaram as mães; foi controlado o tempo de entrevista e a forma de abordagem das mães e, posteriormente, foram discutidas as questões de postura ética, tom de voz e dúvidas em relação às questões do formulário.

Os dados foram agrupados de acordo com as características de semelhança, analisados segundo índices absolutos e percentuais.

Considerou-se como variável dependente o tipo de alimentação oferecida ao bebê nos primeiros seis meses de vida, utilizando-se como embasamento para referir termos e definições sobre aleitamento materno os preconizados pela OMS,10 em 1991, como segue:

Amamentação exclusiva: aleitamento materno como único alimento, e também o lactente podendo receber vitaminas, minerais ou medicamentos;

Amamentação predominante: aleitamento materno mais água, chás, sucos, soro de rehidratação oral;

Amamentação completa: amamentação exclusiva mais amamentação predominante;

Amamentação complementada: leite materno e complemento alimentar semi-sólido ou sólido;

Amamentação: o bebê recebe leite materno, e não importa o consumo de qualquer complemento, lácteo ou não.

Esclarece-se que no presente trabalho, após a coleta dos dados essa terminologia foi adaptada para uma melhor compreensão, a saber:

Aleitamento materno exclusivo (AME): crianças que recebem somente leite materno, sem água, nem chás ou sucos;

Aleitamento materno + outros alimentos
$(\mathrm{AM}+\mathrm{OA})$ : crianças que recebem leite materno de forma predominante, mas também água, chás ou sucos;

Aleitamento materno + artificial (AMA): crianças que recebem leite materno, mas também outros tipos de leite, associados ou não à água, chás, sucos ou cereais.

Neste estudo, utilizou-se também o termo Aleitamento artificial (AA) para as crianças que não receberam leite humano.

As variáveis independentes estudadas foram aquelas relacionadas às condições sócio-demográficas da mãe, e as relacionadas à mãe e ao bebê sobre a gestação, parto e puerpério.

\section{Resultados}

Os resultados são apresentados considerando-se a caracterização das mães das crianças estudadas (faixa etária, estado civil, escolaridade e renda familiar) e segundo ocorrências durante a fase gestacional e puerperal, no que concerne aos aspectos relativos à promoção, incentivo e manutenção do aleitamento natural exclusivo.

Nas Tabelas 1 e 2 são mostrados os aspectos demográficos e sociais das mães das crianças. Verifica-se que apenas $45(21,9 \%)$ crianças não foram amamentadas e que $99(48,3 \%)$ receberam aleitamento materno exclusivo nos primeiros seis meses de vida. Tendo por referência a idade das mães, observa-se que a maioria, $144(70,2 \%)$ tinha idade entre 15 e 31 anos. Destas, 37 forneceram AA e 85 forneceram AME.

Quando se refere ao estado civil, nota-se nessa tabela que $165(80,5 \%)$ mães eram casadas ou tinha união consensual. Dessas, apenas $32(19,4 \%)$ praticaram alimentação artificial, enquanto 133 $(80,6 \%)$ amamentaram, sendo que $85(51,5 \%)$ por aleitamento natural exclusivo nos primeiros seis meses de vida do seu bebê.

A relação entre o tipo de alimentação fornecida às crianças e o grau de escolaridade das mães e renda familiar estão apresentadas na Tabela 2, na qual se verifica que das 76 mães que tinham apenas o ensino fundamental incompleto, 14 deram alimentação artificial ao seu bebê, enquanto as restantes amamentaram, sendo 38 de forma exclusiva até o sexto mês de vida. Dentre as 73 mães com escolaridade entre ensino médio completo e parte do superior (56 mães) ou superior (17 mães) amamentaram. Destas, 31 praticaram aleitamento materno natural exclusivo pelo menos nos seis primeiros meses de vida do seu bebê. 
Tabela 1

Distribuição das mães, segundo a faixa etária e estado civil e o tipo de alimentação do bebê nos primeiros seis meses de vida.

Votuporanga, São Paulo, 2001

\begin{tabular}{|c|c|c|c|c|c|c|c|c|c|c|}
\hline \multirow[t]{3}{*}{ Tipo de alimentação } & \multicolumn{5}{|c|}{ Idade (anos) } & \multicolumn{5}{|c|}{ União civil } \\
\hline & \multirow[t]{2}{*}{15 a 23} & \multirow[t]{2}{*}{24 a 31} & \multirow[t]{2}{*}{32 a 43} & \multicolumn{2}{|c|}{ Total } & \multirow{2}{*}{$\begin{array}{l}\text { Casada/união } \\
\text { consensual }\end{array}$} & \multirow[t]{2}{*}{ Solteira } & \multirow[t]{2}{*}{ Separada } & \multicolumn{2}{|c|}{ Total } \\
\hline & & & & $n$ & $\%$ & & & & $\mathrm{n}$ & $\%$ \\
\hline AA & 23 & 14 & 8 & 45 & 21,9 & 32 & 13 & - & 45 & 21,9 \\
\hline AMA & 6 & 5 & 8 & 19 & 9,3 & 16 & 3 & - & 19 & 9,3 \\
\hline AMOA & 20 & 14 & 8 & 42 & 20,5 & 32 & 8 & 2 & 42 & 20,5 \\
\hline Total & 87 & 80 & 38 & 205 & 100,0 & 165 & 35 & 5 & 205 & 100,0 \\
\hline
\end{tabular}

$\mathrm{AA}=$ Aleitamento artificial; $\mathrm{AME}^{*}=$ Aleitamento materno exclusivo; $\mathrm{AMA}=$ Aleitamento materno + artificial; $\mathrm{AMOA}=\mathrm{Aleitamento}$ materno e outros alimentos.

Tabela 2

Distribuição das mães, segundo o grau de escolaridade e renda familiar e o tipo de alimentação fornecida ao bebê nos primeiros seis meses de vida. Votuporanga, São Paulo, 2001.

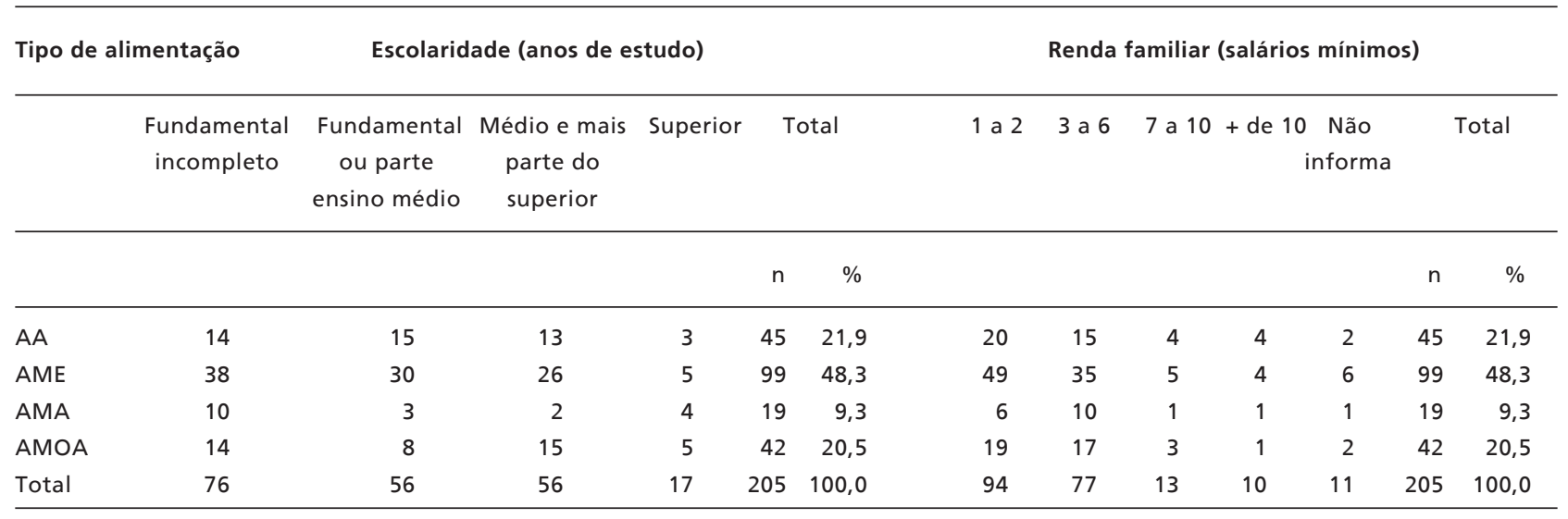

$\mathrm{AA}=$-Aleitamento artificial; $\mathrm{AME}=$ Aleitamento materno exclusivo; $\mathrm{AMA}=$ Aleitamento materno + artificial; $\mathrm{AMOA}=\mathrm{Aleitamento}$ materno e outros alimentos

Comparando-se a renda familiar com o tipo de alimento fornecido ao bebê no primeiro semestre de vida, observa-se que das 94 mães com renda entre um e dois salários mínimos, 20 não amamentaram e 49 praticaram aleitamento materno exclusivo. Já entre as 23 mães com maior renda familiar mensal, isto é, sete ou mais salários mínimos, 8 não amamentaram e 9 amamentaram de forma exclusiva.

Em relação à assistência profissional recebida pela mãe durante a fase gestacional, nota-se, na Tabela 3, que dentre as 203 mulheres que fizeram pré-natal, 76 não receberam orientações sobre a amamentação nessas consultas e ainda assim, 37 amamentaram de forma exclusiva. Já entre as 127 que receberam informações sobre a amamentação no pré-natal, só 25 não amamentaram, enquanto 62 amamentaram de forma exclusiva nos primeiros seis meses de vida do bebê.

No que concerne ao tipo de parto, verifica-se que $152(74,1 \%)$ foram cesáreas, destacando-se que, neste estudo, o tipo de parto não influenciou a condição do bebê receber ou não aleitamento materno.

Pela Tabela 4, pode-se destacar: a) dentre as 46 mães que foram influenciadas quanto à alimentação 
Atendimento pré-natal, parto e tipo de alimentação fornecida ao bebê. Votuporanga, 2001.

\begin{tabular}{|c|c|c|c|c|c|c|c|c|c|}
\hline \multirow[t]{3}{*}{ Tipo de alimentação } & \multicolumn{5}{|c|}{ Atendimento pré-natal } & \multicolumn{4}{|c|}{ Tipo de parto } \\
\hline & \multirow[t]{2}{*}{ Não fez } & \multirow{2}{*}{$\begin{array}{c}\text { Fez sem } \\
\text { orientação }\end{array}$} & \multirow{2}{*}{$\begin{array}{c}\text { Fez com } \\
\text { orientação }\end{array}$} & \multicolumn{2}{|c|}{ Total } & \multirow[t]{2}{*}{ Normal } & \multirow[t]{2}{*}{ Cesárea } & \multicolumn{2}{|c|}{ Total } \\
\hline & & & & $\mathrm{n}$ & $\%$ & & & $\mathrm{n}$ & $\%$ \\
\hline AA & - & 20 & 25 & 45 & 21,9 & 11 & 34 & 45 & 21,9 \\
\hline AME & - & 37 & 62 & 99 & 48,3 & 25 & 74 & 99 & 48,3 \\
\hline AMA & 1 & 8 & 10 & 19 & 9,3 & 5 & 14 & 19 & 9,3 \\
\hline AMOA & 1 & 11 & 30 & 42 & 20,5 & 12 & 30 & 42 & 20,5 \\
\hline Total & 2 & 76 & 127 & 205 & 100,0 & 53 & 152 & 205 & 100,0 \\
\hline
\end{tabular}

$\mathrm{AA}=$-Aleitamento artificial; $\mathrm{AME}=$ Aleitamento materno exclusivo; $\mathrm{AMA}=$ Aleitamento materno + artificial; $\mathrm{AMOA}=\mathrm{Aleitamento}$ materno e outros alimentos.

do bebê, a maioria, (30) teve influência de outras pessoas, seguidas das próprias mães (10), sendo que para 12 sugeriu-se AA e para $21 \mathrm{AME}$; b) quanto à freqüência das mamadas, $126(62,1 \%)$ relatraram ter oferecido alimentação, principalmente nas primeiras semanas pós-parto, de 2/2 horas (68 mães) ou 3/3

Tabela 4

Distribuição das mães segundo ocorrências no puerpério em relação ao tipo de alimento fornecido ao bebê. Votuporanga, São Paulo, 2001.

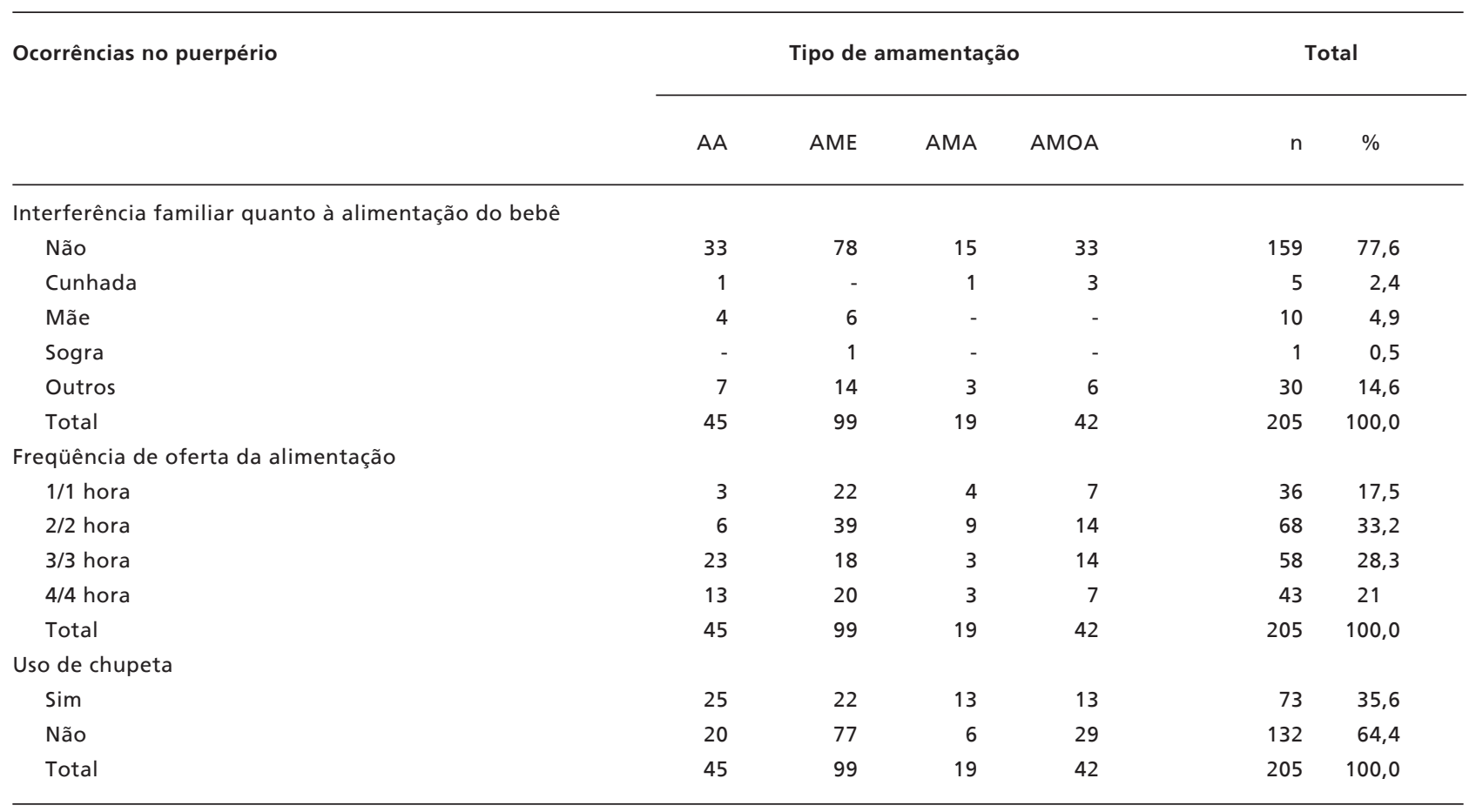

$\mathrm{AA}=$-Aleitamento artificial; $\mathrm{AME}=$ Aleitamento materno exclusivo; $\mathrm{AMA}=$ Aleitamento materno + artificial; $\mathrm{AMOA}=\mathrm{Aleitamento}$ materno e outros alimentos. 
horas (58 mães), sendo que nesse período, 57 realizaram AME; c) quanto ao uso da chupeta, a maioria (132) informou que não fez uso, sendo que destas, 20 praticaram AA e 77 realizaram AME. Dentre as 73 crianças que usaram chupeta, 25 não foram amamentadas e 22 receberam AME.

\section{Discussão}

No que se refere ao tipo de alimentação indicado para crianças de 0 à 6 meses de idade, foi possível observar que a alimentação oferecida à maioria das crianças não era adequada, visto que a OMS preconiza leite materno sem a introdução de outros tipos de alimentos nessa fase da vida. 1

$\mathrm{Na}$ literatura especializada são apresentadas várias causas para o desmame precoce. Em relação à mãe, na decisão de amamentar ou não exclusivamente o seu bebê, os autores apontam para alguns fatores que devem ser considerados, principalmente os socioeconômicos e culturais. 7,8

Assim, no presente trabalho, observou-se que a maior parte das mães tinha idade entre 15 e 31 anos, eram casadas ou viviam em união consensual, possuiam escolaridade em nível fundamental incompleto e rendimento familiar de um a seis salários mínimos.

Em relação à idade materna, tais dados são diferentes quando comparados com outras pesquisas que tratam desse assunto, pois em recentes estudos sobre alimentação nos primeiros anos de vida da criança, as amostras aleatórias da população revelam que a maioria das mães estão na faixa entre 20 e 34 anos, sendo que desse grupo as de maior idade geralmente amamentam seus filhos e por um período de maior duração do que as mães adolescentes ou mais jovens. ${ }^{11,12}$

A predominância do estado civil observado, é o modelo de família composto por pai, mãe e filho(s), afirmando a cultura da necessidade da figura masculina no contexto familiar.

Nos últimos anos uma atenção maior tem sido voltada à associação entre aleitamento materno e fatores psicossociais; há evidências que sugerem a importante participação do pai no apoio às mães durante a amamentação, as influências recebidas de pessoas do convívio social com a intenção de duração do aleitamento e a sua consistência entre a teoria e a prática. ${ }^{13-15}$

Por outro lado, muitas mulheres vem assumindo o papel de chefes de família, somando-se ao de mãe, voltada para as questões domésticas como saúde e educação dos filhos nos mais variados arranjos familiares de nossa sociedade. Entre outros, talvez o principal motivo tenha sido a necessidade financeira que levou a mulher a trabalhar fora de seu lar.

Contextualizando-se com a amamentação, estudos brasileiros evidenciaram a prevalência maior de AME nas mulheres mais instruídas: 0,6 meses nas crianças cujas mães tinham pouca ou nenhuma escolaridade e de 1,2 meses com mães mais instruídas. ${ }^{7-9,13-16}$

Outros trabalhos verificaram que a amamentação reage positivamente a um melhor nível de renda familiar nos primeiros meses de vida. Os dados obtidos nessas pesquisas revelaram uma média de 3,9 meses de amamentação exclusiva entre crianças, cujas famílias ganhavam até um salário mínimo e de 5,3 meses para aquelas com renda familiar maior que 10 salários mínimos. 17,18

$\mathrm{Na}$ presente investigação, outro fator que pode ter influenciado na prática do aleitamento exclusivo foi a assistência profissional recebida pela mãe durante a fase gestacional.

Assim, foi verificado que somente duas mulheres não fizeram pré-natal e que nenhuma delas praticou aleitamento materno artificial. E das duzentas e três que realizaram pré-natal, $62,6 \%$ receberam orientações sobre a amamentação durante as consultas, sendo que a maioria praticou o aleitamento natural de forma exclusiva.

Em estudo realizado em uma maternidade-escola do Rio de Janeiro, constatou-se que 53,3\% das mulheres disseram ter recebido informações sobre aleitamento materno no pré-natal; porém observouse também que essas informações não são plenamente fixadas por elas. Esses dados são preocupantes pois é possível considerar que esse tipo de atendimento não seja suficiente para a fixação de um número tão grande de informações. Sugere-se um acompanhamento pós-parto e durante todo o período do aleitamento, para que as mulheres possam ser orientadas e estimuladas à prática do AME. 19

A partir disso, pesquisou-se se o tipo de parto tem alguma influência sobre a mãe na escolha do tipo alimentação a ser oferecida ao bebê nos primeiros seis meses de vida. Assim, percebeu-se que os resultados verificados foram diferentes dos encontrados nos poucos estudos brasileiros existentes sobre o assunto, pois em pesquisa realizada em município do interior paulista, as crianças nascidas por cesarianas apresentaram maior risco para o desmame. 20

Além de fatores socioeconômicos, os problemas relacionados aos hábitos e cultura materna, comumente são relatados como influenciadores na prática da amamentação. Porém, os dados obtidos revelaram 
que a maioria das mulheres participantes do presente estudo não apresentou problemas que pudessem influenciar diretamente na amamentação, situação que normalmente levaria à interferência de pessoas do âmbito familiar e social.

Para Almeida e Novak ${ }^{21}$ a caracterização da amamentação como um híbrido natureza-cultura procura apresentar uma abordagem da amamentação como um reflexo de determinantes biológicos e condicionantes sociais, econômicos, políticos e culturais, buscando não reduzir a espécie humana à condição de um mamífero qualquer, biológica e socialmente falando.

Dentre os aspectos biológicos da amamentação, os estudiosos afirmam que o aleitamento materno não deve produzir dor, sendo essa a principal causa da maioria dos problemas de amamentação, pois interfere com o reflexo de ejeção do leite. Em conseqüência de o bebê não conseguir mamar, a mãe sente-se angustiada, o que inibe ainda mais a ejeção láctea, podendo conduzir ao fracasso da amamentação. 22

Relata-se um risco maior para o desmame precoce quando são apresentadas dificuldades do tipo ingurgitamento mamário, fissuras, problemas com o mamilo e mastite nos primeiros dias, havendo necessidade constante do estímulo e apoio a essas mães, principalmente de profissionais devidamente capacitados. 23

Também é comum as mães adotarem esquema de horários pré-estabelecidos no oferecimento da alimentação para a criança, talvez pela necessidade de conciliação com outros afazeres do lar ou até mesmo para descansar.23 Nesse contexto, no presente trabalho observou-se que os intervalos de duas a três horas foram os escolhidos pela maioria delas.

O esvaziamento gástrico do lactente, alimentado somente com leite materno, é de aproximadamente uma hora e meia, sendo possível que ele volte a sentir fome após esse período. Recomenda-se, ainda, amamentar pelo sistema de livre demanda, ou seja, quando o lactente solicita, e não com um horário préestabelecido. O número de mamadas no período de recém-nato deve oscilar entre 7 e 12, nas 24 horas, incluindo-se pelo menos uma mamada durante a noite. 22

Considera-se conveniente que o lactente esvazie uma mama antes de se lhe oferecer a outra, de forma que ele receba o leite do final da mamada, porque contém um maior teor de gordura. 22

Um outro fator que comumente interfere no sucesso da amamentação é a introdução de bicos artificiais. Alguns autores afirmam que a criança que não usa outros tipos de bicos tem 1,87 vezes mais possibilidades de ter AME, pois o movimento efetuado pelo lactente ao succionar uma chupeta é diferente do que ele exerce ao mamar. Habituando-se com a chupeta, o lactente empurrará com sua língua o mamilo para fora da boca, em lugar de comprimílo contra o palato, não obtendo leite e provocando dor à mãe. 22

Sobre o uso de chupeta, em recente estudo desenvolvido em Porto Alegre, Rio Grande do Sul, foi verificado que a incidência de desmame entre o primeiro e o sexto mês, nas crianças ainda amamentadas no final do primeiro mês, foi de $22,4 \%$ para aquelas não usuárias de chupeta, e de $50,8 \%$ para as usuárias e, quase 2/3 das usuárias de chupeta deixaram de ser amamentadas exclusivamente até o final do segundo mês e entre as não usuárias, com o índice de $45 \% .24$

Ainda, Lamounier25 alerta para que o uso de chupeta deve ser percebido pelos profissionais da saúde como um marcador de dificuldades do aleitamento materno e, nesse caso, o mais importante talvez não seja somente a retirada da chupeta, e sim a tentativa de detectar e solucionar esses problemas.

$\mathrm{Na}$ presente pesquisa, embora os resultados encontrados estejam abaixo do recomendado, verificou-se que a maioria das crianças foi amamentada de forma exclusiva, e quando comparados com dados encontrados em estudos semelhantes, pode-se observar uma adesão satisfatória à prática da amamentação no município.

Vários são os fatores que podem explicar essa tendência. Talvez o mais relevante seja a existência de grupos de promoção e apoio ao aleitamento materno nas Unidades Básicas de Saúde implantadas desde 1994, onde as reuniões semanais proporcionam o encontro das mães com uma equipe multiprofissional disposta a auxiliá-las na tarefa da amamentação; assim também o acompanhamento e avaliação do crescimento e desenvolvimento do bebê.

Corrobora essa hipótese uma ampla pesquisa realizada sobre aleitamento materno exclusivo no estado de São Paulo: entre outras conclusões, o autor informa que quanto maior o número de ações implementadas pelo município, maiores serão as chances do aleitamento ser exclusivo até o sexto mês. 26

São oito as ações analisadas no referido estudo, e que fazem parte do "Programa Nacional de Incentivo ao Aleitamento Materno" implantado pelo Ministério da Saúde: treinamento dos profissionais envolvidos em aleitamento materno; existência de "Hospital Amigo da Criança"; Bancos de Leite Humano; realização de atividades na "Semana de A- 
mamentação"; políticas públicas de aleitamento (municipais); monitorização da "Norma Brasileira de Comercialização de Alimentos para Lactentes"; equipe multiprofissional que trabalhe na promoção do aleitamento e atividades de pesquisa sobre aleitamento. 26

Desse modo, sugerimos que os dados apresen-

\section{Agradecimentos}

À Secretaria Municipal de Saúde de Votuporanga, São Paulo, pela compreensão e cessão do espaço, aos acadêmicos do curso de enfermagem pela seriedade e dedicação e ao Centro Universitário de Votuporanga (UNIFEV) pelo apoio e incentivo à pesquisa.

\section{Referências}

1. World Health Organization. The World Health Organization's infant-feeding recommendation. Bull World Health Organ 1995; 73: 165-74.

2. Scariati PD, Grummer-Dtrawn LM, Beck Fein AS . Longitudinal analysis of infant and extent of breastfeeding in the United States. Pediatrics 1997; 99: 5-11.

3. César JA, Victora CG, Barros FC, Santos IS e Flores JA. Impact of breastfeeding on admission for pneumonia during postneonatal period in Brazil: nested case-control study. $\mathrm{Br}$ Med J 1999; 318: 1316-20.

4. American Academy of Pediatrics. Breastfeeding and the use of human milk. Pediatrics 1997; 100: 1035-9.

5. Lamounier JA. Experiência iniciativa Hospital Amigo da Criança. Rev Ass Med Bras 1998; 44: 319-24.

6. Réa MF. Reflexões sobre a amamentação no Brasil: de como passamos a 10 meses de duração. Cad Saúde Pública 2003; 19 (Supl 1): S37-S45.

7. Montrone VG, Arantes CIS. Prevalência do aleitamento materno na cidade de São Carlos, São Paulo. J Pediatr (Rio J) $2000 ; 76: 138-42$.

8. Kummer SC, Giugliani ERJ, Susin LO, Folletto JI, Lermen NR, Wu VYJ, Santos L, Caetano MB. Evolução do padrão de aleitamento materno. Rev Saúde Pública 1999; 34: 1438 .

9. Venâncio SI, Escuder MML, Kitoko P, Réa MF, Monteiro CA. Freqüência e determinantes do aleitamento materno em municípios do Estado de São Paulo. Rev Saúde Pública 2002; 36: 313-8.

10. OMS (Organização Mundial de Saúde). Indicadores para evaluar las prácticas de lactancia materna. Genebra; 1991.

11. Gigante DP, Victora CG, Barros FC. Nutrição materna e duração da amamentação em uma coorte de nascimento de Pelotas, RS. Rev Saúde Pública 2000; 34: 259-65.

12. Scott JA, Binns CW. Factors associated with the initiation and duration of breastfeeding: a review of the literature tados no presente estudo levem a outras investigações dessa natureza, colaborando para o sucesso da amamentação, e conseqüente diminuição nos índices de morbi-mortalidade infantil. E possam também, a partir disso, possibilitar a elaboração de planos de intervenção visando o aleitamento materno exclusivo.
[Breastfeeding review]. J Nur Mother Ass Aust 1999; 7: 516.

13. Bueno MB, Souza JMP, Souza SB, Paz SMRS, Gimeno SGA, Siquiera AAF. Riscos associados ao processo de desmame entre crianças nascidas em hospital universitário de São Paulo, entre 1998 e 1999: estudo de coorte prospectivo do primeiro ano de vida. Cad Saúde Pública 2003; 19 : 1453-60.

14. Ramos CV, Almeida JAG. Alegações maternas para o desmame: estudo qualitativo. J Pediatr (Rio J) 2003; 79: 385-90.

15. Ichisato SMT, Shimo AKK. Revisitando o desmame precoce através de recortes da história. Rev Latinoam Enf 2002; 10: 578-85.

16. Passos MC, Lamounier JA, Silva CAM, Freitas SN, Baudson MFR. Práticas da amamentação no município de Ouro Preto, MG, Brasil. Rev Saúde Pública 2000; 34: 61722.

17. Kitoko PM, Réa MF, Venancio SI, Vasconcelos ACCP, Santos EKA, Monteiro CA. Situação do aleitamento materno em duas capitais brasileiras: uma análise comparada. Cad Saúde Pública 2000; 16: 1111-9.

18. Hugles R, Cox S. An analysis of breastfeeding initiation in Tasmania by demographic and socioeconomic factors for the period 1981-1995. [Breastfeeding review]. J Nur Mother Ass Aust 1999; 7: 19-22.

19. Pereira GS, Colares LGT, Carmo MGT, Soares EA. Conhecimentos maternos sobre amamentação entre puérperas inscritas em programa de pré-natal. Cad Saúde Pública 2000; 16: 457-66.

20. Figueiredo MG, Sartorelli DS, Zan TAB, Garcia E, Silva LC, Carvalho FLP, Pascotto RC, Macri S, Cardoso MA. Inquérito de avaliação rápida das práticas de alimentação infantil em São José do Rio Preto, São Paulo, Brasil. Cad Saúde Pública 2004; 20: 172-9. 
21. Almeida JAG, Novak FR. Amamentação: um híbrido natureza-cultura. J Pediatr (Rio J) 2004; 80: 119-125.

22. Valdés V, Sánches AP, Labbok M. Manejo clínico da lactação, assistência à nutriz e ao lactente. Rio de Janeiro: Revinter; 1996.

23. Caldeira AP, Goulart EMA. A situação do aleitamento materno em Montes Claros, Minas Gerais: estudo de uma amostra representativa. J Pediatr (Rio J) 2000; 76: 65-71.

24. Soares MEM, Giugliani ERJ, Braun ML, Salgado ACN, Oliveira AP, Aguiar PR. Uso de chupeta e sua relação com o desmame precoce em população de crianças nascidas em Hospital Amigo da Criança. J Pediatr (Rio J) 2003; 79: 30916.

Apresentado em 25 de agosto de 2004

Versão final em 7 de junho de 2005

Aprovado em 12 de agosto de 2005
25. Lamounier JA. O efeito de bicos e chupetas no aleitamento materno. J Pediatr (Rio J) 2003; 79: 284-86.

26. Venâncio SI. Determinantes individuais e contextuais do aleitamento materno exclusivo nos primeiros seis meses de vida em 111 municípios do Estado de São Paulo [tese doutorado] São Paulo: Faculdade de Saúde Pública da Universidade de São Paulo; 2002. 